\title{
Rituels épistolaires dans les lettres des poilus peu et moins lettrés : une analyse contrastive
}

\author{
Stefano Vicari \\ Département de Langues Cultures Modernes,Université de Gênes, Italie \\ stefano.vicari@unige.it
}

\begin{abstract}
Résumé. Dans cet article, on propose une analyse contrastive des rituels épistolaires dans les lettres des poilus selon le niveau d'instruction des scripteurs, des moins aux plus lettrés. Suite à la présentation des remarques méthodologiques, des objectifs et de la constitution du corpus, il s'agira d'exposer le cadre théorique et les recherches déjà menées sur les lettres des scripteurs peu lettrés afin de mieux cerner les enjeux des analyses. Enfin, on passera à l'analyse des formules d'ouverture et de clôture des lettres ainsi que des transitions entre ces parties formulaires et le corps de la lettre, dans le but de dégager les différences et les ressemblances entre les scripteurs peu lettrés et plus lettrés.
\end{abstract}

In this paper we propose a contrastive analysis of the epistolary rituals in the letters of the soldiers of the First World War according to their level of education. After the presentation of the methodology, the objectives and the constitution of the corpus, we will present the theoretical framework and the researches already carried out on the letters of "peu lettrés". Finally, we will analyze the opening and closing formulas of the letters as well as transitions between these parts and the body of the letter, in order to observe differences and similarities between "peu lettrés" and "plus lettrés" writers.

\section{Introduction}

Cet article se pose l'objectif d'analyser les « rituels épistolaires » d'ouverture et de clôture dans un corpus de lettres de poilus plus et moins lettrés et s'appuie principalement sur des études qui ont déjà été menées sur les mêmes types de rituels dans les lettres des poilus peu lettrés (Branca-Rosoff, 2015, Große S., Steuckardt A., Dal Bo B., Sowada L., 2016). L'intérêt de cette étude réside dans le fait que les recherches sur le genre épistolaire se sont davantage centrées soit sur l'écriture littéraire (on peut citer, sans souci d'exhaustivité, 
Jaubert, Siess, etc.) soit sur l'écriture des lettres de la part des peu lettrés qu'ils soient poilus ou non (Branca-Rosoff Sonia, 2015, Pellegrini Florence, 2015, Bellosi et Savini, [2002] 2014, Moreux Bernard, 2001, Bruneton-Governatori Ariane, Moreux Bernard, 1997). Les lettres analysées dans cette étude relèvent de gens ordinaires qui, obligés de s'éloigner de leurs familles pour rejoindre le front, dans des situations précaires et périlleuses, ressentent la nécessité d'écrire pour garder un contact avec l'arrière et avec leurs proches, avec tout un monde qui, suite à l'entrée en guerre, apparaît à leurs yeux comme incommensurable.

\section{Objectifs, corpus et méthodologie}

Cette étude, qui a un caractère exploratoire vue l'extension limitée du corpus, se propose d'analyser les formules d'ouverture et de clôture dans les lettres rédigées par des poilus se situant sur une échelle qui va des moins aux plus scolarisés, afin de comparer l'emploi des formules dans les correspondances. Voici un tableau présentant les identités des scripteurs, leur niveau d'instruction, leur profession avant la guerre, leur origine et leur grade dans l'armée française :

Tableau 1. Données des scripteurs du corpus

\begin{tabular}{|c|c|c|c|c|}
\hline Poilu & $\begin{array}{c}\text { Niveau } \\
\text { d'études }\end{array}$ & Profession & Origine & Grade \\
\hline $\begin{array}{c}\text { Henri } \\
\text { Bénard }\end{array}$ & $\begin{array}{c}\text { Ecole de } \\
\text { Saint-Maixent }\end{array}$ & $\begin{array}{c}\text { capitaine dans } \\
\text { l'armée } \\
\text { (haute } \\
\text { bourgeoisie) }\end{array}$ & Arles & $\begin{array}{c}\text { Officier - } \\
\text { commandant }\end{array}$ \\
\hline $\begin{array}{c}\text { Maurice } \\
\text { Pensuet }\end{array}$ & $\begin{array}{c}\text { certificat } \\
\text { d'études }\end{array}$ & $\begin{array}{c}\text { ? } \\
\text { (petite } \\
\text { bourgeoisie) }\end{array}$ & $\begin{array}{c}\text { Meung-sur- } \\
\text { Loire [Loiret] }\end{array}$ & caporal \\
\hline $\begin{array}{c}\text { Joseph } \\
\text { Papillon }\end{array}$ & $\begin{array}{c}\text { certificat } \\
\text { d'études }\end{array}$ & $\begin{array}{c}\text { bourrelier-sellier } \\
\text { (famille } \\
\text { d'agriculteurs) }\end{array}$ & $\begin{array}{c}\text { Vézelay } \\
\text { (Yonne) }\end{array}$ & soldat d'infanterie \\
\hline $\begin{array}{c}\text { Lucien } \\
\text { Papillon }\end{array}$ & $\begin{array}{c}\text { école primaire } \\
\text { - pas de } \\
\text { certificat }\end{array}$ & $\begin{array}{c}\text { (familleur } \\
\text { d'agriculteurs) }\end{array}$ & $\begin{array}{c}\text { Vézelay } \\
\text { (Yonne) }\end{array}$ & soldat d'infanterie \\
\hline
\end{tabular}

L'on passe de Lucien, qui a fréquenté l'école primaire sans pourtant obtenir son certificat d'études à Henri qui, issu d'une famille de la haute bourgeoisie, est diplômé à l'Ecole pour les sous-officiers de Saint-Maixent, en passant par Joseph et Maurice. S'il est vrai que tant Joseph que Maurice sont titulaires d'un certificat d'études, l'orthographe de Joseph est plus incertaine que celle de Maurice, qui montre quant à lui une bonne maîtrise du code écrit. Pour chaque scripteur ont été analysées 10 lettres par an entre 1914 et 1918. Le fait de choisir les lettres sur plusieurs années permet de se poser la question de savoir si l'écriture épistolaire, qui au fur et à mesure que le temps passe se transforme en une véritable pratique quotidienne, porte les traces de cette routinisation dans l'adoption d'un style formulaire plus massif. C'est du moins ce qui arrive au niveau thématique : les scripteurs soulignent à plusieurs reprises non seulement la monotonie dans la vie des tranchées, mais aussi les retombées que leur quotidien aurait sur le contenu de leurs lettres. Voici un exemple : 
(1) On a si peu de choses à dire ! S'il fallait que je te raconte tout ce que je fais, tout ce que je vois, il y aurait beaucoup à te raconter mais ce serait toujours la même chose. Cette guerre, en effet, se déroulant toujours sur le même terrain, on ne peut décrire de faits sensationnels nouveaux. (Henri, le 30/03/1915)

Or, s'il est vrai que les rituels épistolaires apparaissent comme caractéristiques du genre épistolaire populaire, il me semble que le critère du moment de l'écriture doit être pris en compte dans les analyses afin de vérifier une progression éventuelle dans l'emploi de ces rituels sur l'axe temporel. Le tableau suivant montre plus dans le détail la constitution du corpus :

Tableau 2. Corpus

\begin{tabular}{|c|c|c|c|c|c|c|}
\hline Poilu & 1914 & 1915 & 1916 & 1917 & 1918 & TOT \\
\hline $\begin{array}{c}\text { Henri } \\
\text { Bénard }\end{array}$ & 10 & 10 & 10 & & & 30 \\
\hline $\begin{array}{c}\text { Maurice } \\
\text { Pensuet }\end{array}$ & 10 & 10 & 10 & & & 30 \\
\hline $\begin{array}{c}\text { Joseph } \\
\text { Papillon }\end{array}$ & 10 & 10 & & & & 20 \\
\hline $\begin{array}{c}\text { Lucien } \\
\text { Papillon }\end{array}$ & 1 & 10 & 10 & 10 & 5 & 36 \\
\hline & 31 & 40 & 30 & 10 & 5 & 116 \\
\hline
\end{tabular}

Les expériences de guerre varient sensiblement selon le poilu : pour les deux scripteurs plus lettrés du corpus on n'a pu analyser que les dix lettres des trois premières années de guerre, pour Joseph seulement deux, alors que Lucien est le seul de ces soldats qui a pu rentrer au foyer une fois que la guerre est finie. Le nombre exigu des lettres analysées (116, pour un total de 69.453 mots) confère à cette étude un caractère exploratoire, ce qui n'empêche pas d'identifier des tendances à vérifier éventuellement sur un corpus plus large. Et notamment, le corpus ainsi constitué permet de se poser trois types de questions auxquelles on essayera de répondre même si de manière non définitive : quelle est la relation entre l'emploi de formules et le niveau d'instruction ? Peut-on établir une relation entre l'emploi de formules et le moment de guerre ? Quelles transitions peut-on trouver entre la formule d'ouverture et le corps de la lettre et entre le corps de la lettre et la formule de clôture ? On a adopté la typologie de formules proposée par Rutten et Van der Wal (2013) suivant laquelle, il est possible de distinguer entre " text-type formulae » et « textstructural formulae $»$ : les premières permettent d'identifier le genre auquel appartient le texte, dans ce cas, des lettres (date, terme d'adresse, lieu et formules d'ouverture et de clôture), les secondes jalonnent la progression textuelle et ne sont pas typiques du genre «lettre». En outre, en ce qui concerne les formules d'ouverture, elles ont été également divisées en deux catégories dégagées des travaux de Bellosi et Savini ([2002] 2014), à savoir formules déclaratives et responsives. Les formules déclaratives se trouveraient en ouverture des lettres qui ne constituent pas une réponse à une lettre précédente, ou mieux, qui n'affichent pas leur caractère responsif, alors que les formules responsives seraient employées là où les scripteurs répondent de manière explicite à une lettre, à une carte ou à 
un colis reçu. Voici deux exemples de formule déclarative et responsive telles qu'on peut les retrouver dans les lettres des poilus :

(2) Je vous envoie ce mot par un permissionnaire pour vous faire parvenir plus rapidement de mes nouvelles. Je suis en ce moment à Picquigny, un peu en arrière d'Amiens. (Lucien, le 26/05/1918).

(3) J'ai bien reçu le paquet de chocolat et la lettre. J'en assez pour l'instant, il ne faut pas m'en envoyer avant le 1 Décembre. J'ai bien reçu une carte postale de quelques mots de Vincent, mais je n'ai pas encore vu sa lettre ni le paquet que vous m'annoncez de lui. (Maurice, le 14/11/1914).

Dans le but d'analyser la structure de ces formules et leur enchainement dans le tissu textuel, on a adopté le schéma élaboré par (Große S., Steuckardt A., Dal Bo B., Sowada L., 2016) à partir des lettres des poilus peu lettrés. Les formules déclaratives seraient alors composées «(1) d'une première partie comportant des verbes du type envoyer, écrire suivis de constructions permettant de dénoter l'état de sa santé, et (2) d'une deuxième partie qui exprime le souhait que la santé du (des) destinataire(s) soit bonne » (Ibid., en ligne; ital. des auteures) et les formules responsives seraient constituées «(1) d'une première partie comportant des verbes comme répondre, faire réponse suivis d'un complément d'objet indirect dénotant les lettres des correspondants et leur contenu en termes de santé, (2) d'une deuxième partie dans laquelle le scripteur affirme le bon état de sa propre santé » (Ibid., en ligne; ital. des auteures). Pour ce qui est des formules de clôture, une typologie sera élaborée sur la base des résultats des analyses.

\section{3 « Rituels épistolaires » et lettres des poilus (peu lettrés)}

Comme ces quelques remarques permettent déjà d'entrevoir, la lecture des correspondances des poilus ne va pas sans susciter auprès du lecteur contemporain une certaine impression de répétitivité, voire de monotonie. Un bon nombre de topoï semblent parcourir de manière transversale les lettres des poilus plus et peu lettrés (Luxardo, 2015, Pellegrini, 2015), tout comme celles des soldats italiens engagés dans la même guerre (Gibelli, 1991). C'est bien le quotidien de la vie dans les tranchées qui ressort de ces textes, dans toute sa simplicité, voire banalité et de manière un tant soit peu stéréotypée. Ce caractère réitératif tant dans les routines quotidiennes des scripteurs que dans la façon dont ils la verbalisent est bien montré par l'extrait suivant où, sous la plume ironique de Gaston Olivier, on peut lire :

(4) Que veux-tu je n'ai que cela comme passe-temps et je ne fais que ça. Nous en touchons au régiment qui n'est pas mauvais dans la pipe. Je te raconte bien des histoires de pipes. Changeons, et pour changer : la santé ; elle est excellente comme toujours ${ }^{1}$. (Olivier, le 24/11/14)

La récurrence de certaines thématiques dans des structures syntaxiques semi-figées dans les ouvertures et dans les clôtures des lettres semble caractériser l'écriture épistolaire des peu lettrés, qu'il s'agisse des soldats de la Première Guerre Mondiale (Große S., Steuckardt

\footnotetext{
${ }^{1}$ Je souligne dans tous les exemples analysés. Les exemples sont reproduits sans modifications ou corrections de la part de l'auteur.
} 
A., Dal Bo B., Sowada L., 2016), d'émigrés béarnais (Bruneton-Governatori, Moreux, 1997) ou de scripteurs néerlandais du XVIIème et du XVIIIème siècles (Rutten, Van der Wal, 2013). Ainsi, Bruneton-Governatori et Moreux ont-elles dégagé un «modèle épistolaire populaire » (1997) qui s'adapte bien aux lettres des poilus peu lettrés, comme Große S., Steuckardt A., Dal Bo B., Sowada L. (2016) l'ont montré. C'est à partir de ce constat que les auteurs parlent de "compétence spécifiquement épistolaire » (BrunetonGovernatori, Moreux, 1997 : en ligne) qui côtoie celle proprement linguistique (souvent déficitaire) pour les peu lettrés. Ceux-ci auraient déjà dans la tête un schéma tant au niveau des contenus qu'au niveau de la structure et des moyens langagiers à exploiter, tant il est vrai que, selon ces auteurs, « une partie de ces contenus est présente à leur esprit sous la forme de schémas et d'énoncés en quelque sorte préécrits, de formules donc » (Ibid., en ligne), avec des degrés de figement et de récurrence plutôt variables. Ce «modèle épistolaire populaire »

s'est perpétué, transmis sans doute par la lecture et la mémorisation de lettres, rendant possibles des variations et recréations individuelles. Ce modèle a permis de faire accéder des nouvelles banales, quoiqu'essentielles, au statut d'écrit, doublement sacralisé en tant qu'écrit et porteur de valeur d'échange. (Bruneton-Governatori, Moreux, 1997 : en ligne)

Pour appréhender ces «formules », dont le figement n'est pas total (on ne peut pas parler d'unités phraséologiques stricto sensu), les chercheurs ont adopté plusieurs dénominations permettant de saisir la nature de ces éléments structurant le genre épistolaire peu lettré. Ainsi, trouve-t-on sous la plume de Branca-Rosoff (1990) le terme de «conventions épistolaires» pour appréhender non seulement ce que l'auteure appelle « formules rituelles » (Ibid., p.23) d'ouverture et de clôture, mais aussi certains éléments se situant à la frontière entre sémantique et morphosyntaxe à l'intérieur du corps des lettres. Ce sont bien les termes de « formule » et d' « écriture formulaire » que l'on retrouve dans la plupart des recherches portant sur ce type de discours stéréotypé caractérisant les lettres des peu lettrés (Bruneton-Governatori et Moreux, 1997, Moreux, 2001, Rutten, Van der Wal, 2013, Große S., Steuckardt A., Dal Bo B., Sowada L., 2016), à côté de désignations comme «logiques épistolaires » (Pellegrini, 2015) qui portent davantage sur la réappropriation individuelle de ces formules de la part des scripteurs à partir de micro-variations sémantiques et syntaxiques. Ces énoncés, dont Barthes, comme le rappelle Branca-Rosoff (1990 : 22), proposait un classement en tant qu' « énoncés du code culturel » (Barthes, 1970) en vue d'un programme de stylistique renouvelée, semblent caractériser la culture de l'écriture épistolaire, en dépit des recommandations des Secrétaires (Chartier, 1991, Große, 2011), qui déjà vers la fin du XVII ${ }^{\text {ème }}$ insistaient sur la nécessité de réduire les formules toutes faites afin de reproduire de plus près une conversation orale en face à face. C'est justement cette nature à la fois sociale et culturelle soulignée par Barthes qui est saisie par la dénomination de "rituels épistolaires » proposée par Branca-Rosoff (2015), qui a le mérite d'inscrire l'emploi de ces formules dans une dimension anthropologique plus élargie. Tant il est vrai que le long du XIXème siècle, l'écriture et la lecture des lettres deviennent de véritables pratiques culturelles aussi parmi ceux qui jusque-là écrivaient peu ou rien (Hébrard, 1991 : 284-285). Dans la mesure où «les sociabilités populaires de la lettre sont essentiellement collectives » (Ibid., 288), c'est le statut « intime » de la lettre qui se trouve modifié : elle représente par là un objet autour duquel un véritable rituel social de 
lecture publique s'instaure. Sur la base de ces constats, Bruneton-Governatori et Moreux (1997) expliquent la présence du discours formulaire : les formules permettraient alors de garder le lien avec les pratiques de la communauté d'origine, vu que les lettres sont souvent lues publiquement. Cela dit, la confrontation avec l'écriture de la part de scripteurs qui n'ont pas l'habitude d'écrire, de manière si massive au moins, relève d'un effort non négligeable : l'emploi des formules jouerait donc également une fonction cognitive en permettant de réduire cet effort et, par là, de favoriser l'entrée dans l'écrit (Rutten, Van der Wal, 2012, Große S., Steuckardt A., Dal Bo B., Sowada L., 2016). Du point de vue strictement linguistique et textuel, ces formules se présentent sous la forme d'énoncés dont l'effet de répétitivité est créé tant par les thématiques que par des constructions grammaticales et syntaxiques récurrentes, sans que l'on puisse parler de figement, ni lexical ni syntaxique ${ }^{2}$. Il suffit de prendre en compte les critères sur lesquels repose le degré de figement qui ont permis aux auteurs cités d'identifier ces «formules» pour se rendre compte des nombreuses variations micro structurelles caractérisant les styles d'écriture des scripteurs. Il semble en effet que de l'ensemble de ces études on peut dégager trois critères principaux relevant respectivement de la structuration textuelle, de la sémantique et de la syntaxe. Pour ce qui est de la structure de la lettre, ces énoncés encadrent le corps de la lettre : ils se trouvent en ouverture et en clôture, ce qui n'exclut pas leur présence à l'intérieur de la partie considérée comme la plus individuelle :

(5) Pour la couronne à ce pauvre Joseph, je sais bien que Marthe fera tout son possible pour que ce soit fait. Comme je vous l'ai écrit sur une carte, j'ai passé le $\mathrm{I}^{\circ}$ janvier avec $\mathrm{M}$. Combes. Je suis toujours en bonne santé ainsi que les amis qui vous envoient le bonjour. Nous remontons aux tranchées aprèsdemain. Depuis 17 mois que j'ai mon pantalon rouge, je l'ai enfin remplacé ce matin par un autre en velours - tout neuf bien entendu [...]. (Maurice, le 03/01/1916)

Les topoï de la santé et des compliments sont alors insérés au milieu du corps de la lettre, là où les scripteurs sont susceptibles de se livrer à un récit plus intime et personnel.

Si ces rituels (socio)épistolaires caractérisent bien les correspondances des poilus peu lettrés, qu'en est-il des lettres des poilus plus lettrés ? Comme l'affirment Rutten et Van der Wal (2013), l'emploi des formules par les scripteurs plus lettrés serait moins important. En effet, deux sortes d'explications peuvent être identifiées. La première raison tient à la fonction principale remplie par ces formules : les scripteurs plus lettrés, ayant déjà une certaine familiarité avec l'écrit avant l'entrée en guerre, ont sans doute moins de difficulté à traduire à l'écrit leurs pensées. La seconde raison, liée à la précédente, réside dans l'influence des manuels scolaires : si déjà vers la fin du XVIIème siècle, les secrétaires destinés aux classes aristocrates et bourgeoises recommandaient de réduire les formules et le style cérémonieux en faveur d'un style plus naturel, sur le modèle des lettres de Mme de Sévigné (Große, 2011), les manuels de l'école de Ferry, fréquentée d'ailleurs par la plupart des poilus au front (selon le Compte rendu sur le recrutement de l'armée pendant 1905 du Ministère de la Guerre en 1906) insistent sur tout refus de mots cérémonieux et sur la

\footnotetext{
${ }^{2}$ Cela rend d'ailleurs difficile leur traitement en TAL (Große S., Steuckardt A., Dal Bo B., Sowada L., 2016).
} 
préférence qu'il faut accorder à la sincérité du sentiment dans la rédaction de la lettre (Branca-Rosoff, 2015).

\section{Quelques données quantitatives}

Avant d'entrer dans le détail des analyses qualitatives, des données quantitatives permettront de se rendre compte de l'ampleur du discours formulaire dans un corpus hétérogène du point de vue de la maîtrise du code écrit des scripteurs et par là d'avancer quelques hypothèses.

Tableau 3. Nombre de formules d'ouverture par scripteur

\begin{tabular}{|c|c|c|c|c|c|c|c|c|}
\hline Scripteurs & $\begin{array}{c}\text { Tot } \\
\text { lettr } \\
\text { es }\end{array}$ & $\begin{array}{c}191 \\
4\end{array}$ & $\begin{array}{c}191 \\
5\end{array}$ & $\begin{array}{c}191 \\
6\end{array}$ & $\begin{array}{c}191 \\
7\end{array}$ & $\begin{array}{c}191 \\
8\end{array}$ & $\begin{array}{c}\text { Tot } \\
\text { formul } \\
\text { es }\end{array}$ & $\%$ \\
\hline Henri Bénard & 30 & $2 / 10$ & $3 / 10$ & $2 / 10$ & & & 7 & $\begin{array}{c}23 \\
\%\end{array}$ \\
\hline $\begin{array}{c}\text { Maurice } \\
\text { Pensuet }\end{array}$ & 30 & $6 / 10$ & $2 / 10$ & $6 / 10$ & & & 14 & $\begin{array}{c}47 \\
\%\end{array}$ \\
\hline $\begin{array}{c}\text { Joseph } \\
\text { Papillon }\end{array}$ & 20 & $8 / 10$ & $9 / 10$ & & & & 17 & $\begin{array}{c}85 \\
\%\end{array}$ \\
\hline $\begin{array}{c}\text { Lucien } \\
\text { Papillon }\end{array}$ & 36 & $0 / 1$ & $6 / 10$ & $7 / 10$ & $8 / 10$ & $4 / 5$ & 25 & $\begin{array}{c}78 \\
\%\end{array}$ \\
\hline
\end{tabular}

La plupart des formules d'ouverture se concentrent chez les deux scripteurs les moins lettrés du corpus, ce qui ne fait que confirmer le fait que l'une des fonctions des formules est constituée par la réduction de l'effort d'écriture. Il serait donc normal que les scripteurs avec un niveau d'instruction supérieur ne ressentent pas trop le besoin de recourir aux formules pour entrer dans l'écrit. Cela dit, les différences ne sont pas si nettes que l'on imaginait au début entre scripteurs peu lettrés et scripteurs plus lettrés : dans à peu près la moitié des lettres de Maurice l'on retrouve en effet des formules d'ouverture alors que leur nombre diminue sensiblement seulement chez Henri (dont la présence des formules caractérise à peu près un quart des lettres). Les données sur les formules de clôture contribuent de manière encore plus évidente à réduire les différences :

Tableau 4. Nombre de formules de clôture par scripteur

\begin{tabular}{|c|c|c|c|c|c|c|c|c|}
\hline & $\begin{array}{c}\text { Tot } \\
\text { lettres }\end{array}$ & 1914 & 1915 & 1916 & 1917 & 1918 & $\begin{array}{c}\text { Tot } \\
\text { formules }\end{array}$ & $\%$ \\
\hline $\begin{array}{c}\text { Bénard } \\
\text { Henri }\end{array}$ & 30 & $9 / 10$ & $8 / 10$ & $6 / 10$ & & & 23 & $77 \%$ \\
\hline $\begin{array}{c}\text { Maurice } \\
\text { Pensuet }\end{array}$ & 30 & & $9 / 10$ & $10 / 10$ & $10 / 10$ & & 29 & $97 \%$ \\
\hline $\begin{array}{c}\text { Joseph } \\
\text { Papillon }\end{array}$ & 20 & $4 / 10$ & $1 / 10$ & & & & 5 & $30 \%$ \\
\hline $\begin{array}{c}\text { Lucien } \\
\text { Papillon }\end{array}$ & 36 & $0 / 1$ & $3 / 10$ & $3 / 10$ & $1 / 10$ & $3 / 5$ & 10 & $3 \%$ \\
\hline
\end{tabular}


Les tableaux montrent qu'il n'y aurait aucune relation entre l'emploi des formules et le moment de l'expérience de guerre : il ne semble pas que la vie monotone dans les tranchées soit à l'origine d'une plus grande stéréotypisation des textes. Ces chiffres montrent non seulement que les scripteurs les plus lettrés emploient les formules de clôture dans leurs lettres, mais aussi qu'ils y recourent de manière plus massive que leurs camarades moins lettrés. L'hypothèse selon laquelle le discours formulaire caractérise davantage les correspondances des peu lettrés (Rutten et Van der Wal, 2013) ne semble confirmée que partiellement (elle est plus valable pour les ouvertures que pour les clôtures) et pourrait du moins être nuancée par le biais d'une analyse qualitative plus fine.

\section{Les ouvertures}

Dans cette section, les différents types d'ouverture des lettres seront présentées à partir des cas où les formules sont absentes pour passer ensuite à la comparaison des formules entre les scripteurs peu et plus lettrés.

\subsection{Absence de formule}

Comme l'ont déjà remarqué Große S., Steuckardt A., Dal Bo B., Sowada L. (2016), pour les peu lettrés, l'absence de la formule se justifie volontiers par l'urgence du scripteur de communiquer à son destinataire une nouvelle importante, comme ces exemples le montrent :

(6) Mon cher papa, ma chère maman, Ça y est, c'est le grand coup. (Maurice, le 30/04/1915)

(7) Mon cher papa, ma chère maman,

Une nouvelle. Le PDD quitte demain matin Le Chatelier pour se transporter pédibus jambum à Evres $23 \mathrm{~km}$ '. Qu'est-ce qui nous attend là-bas ? Mystère. (Maurice, le 04/01/1916)

(8) Chers parents,

On nous a vivement descendu de la Somme en carnionautos. Depuis 2 jours, nous sommes [...](Joseph, Le 2/06/1918)

(9) Chers parents,

Je suis été operré jeudi, je n'ai pas souffer. Ça c'est trais bien passé. Je suis été andormis. (Lucien, le 12/02/1916)

Dans tous ces cas, la formule d'adresse est aussitôt suivie par une nouvelle ressentie comme importante par les scripteurs, qu'ils soient peu lettrés, comme Joseph ou plus lettrés, comme Maurice. Il s'agit presque toujours de communiquer une action, un changement d'état qui se produit au front, là où la monotonie semble dominer la plupart du temps. Ce caractère d'urgence de la nouvelle est visible dans les moyens langagiers adoptés par les scripteurs. Ainsi, dans le premier exemple, trouve-t-on la locution «ça y est », typique d'ailleurs de l'oral courant, qui signale qu'un événement prévu est en train de se réaliser et rompt l'uniformité de la vie dans les tranchées ; dans le deuxième, c'est l'énoncé 
nominal «Une nouvelle » qui introduit l'information suscitant une certaine inquiétude auprès du scripteur dénoncée par la question rhétorique et la réponse plutôt troublante qui suivent.

Il peut arriver aussi, comme dans (8), que le scripteur doive rassurer le plus vite possible ses proches sur l'état de santé ; l'urgence s'explique là sans doute par l'importance de la nouvelle à communiquer: Lucien a été gravement blessé et il a été opéré, il s'empresse alors de tranquilliser ses parents.

Chez Henri, le capitaine de l'armée ayant fréquenté l'Eole des sous-officiers, l'absence de formule ne s'accompagne pas du besoin de communiquer avec urgence une nouvelle. Généralement, dans ses lettres, les formules d'adresse sont suivies d'un déictique temporel, spatial ou personnel permettant d'entrer directement dans le corps de la lettre :

(10) Ma chère Anine,

Demain soir, nous aurons fini nos huit jours de tranchées et nous allons au repos huit jours dans un village évacué. Aujourd'hui nous avons été bombardés sérieusement (Henri, le 16/01/1916)

\subsection{Typologie des formules d'ouverture}

Pour l'analyse des formules d'ouverture, on a opté pour une distinction fonctionnelle entre formules déclaratives et responsives (Bellosi et Savini ([2002] 2014) et pour une distinction thématique, identifiée par Große S., Steuckardt A., Dal Bo B., Sowada L. (2016) pour les lettres des peu lettrés, entre les formules qui portent sur la mention du courrier et celles qui portent sur la santé. Voici un tableau récapitulatif des résultats issus du corpus analysé :

Tableau 5. Typologie des formules d'ouverture

\begin{tabular}{|c|c|c|c|c|}
\hline & \multicolumn{2}{|c|}{ Déclarative } & \multicolumn{2}{c|}{ Responsive } \\
\hline & Santé & courrier & Santé & courrier \\
\hline Henri Bénard & 0 & 0 & 0 & 4 \\
\hline Maurice Pensuet & 2 & 4 & 0 & 10 \\
\hline Joseph Papillon & 3 & 4 & 4 & 15 \\
\hline Lucien Papillon & 1 & 4 & 2 & 16 \\
\hline Tot & $17(17 \%)$ & $19(19 \%)$ & $6(6 \%)$ & $64(64 \%)$ \\
\hline
\end{tabular}

\subsubsection{Les formules d'ouverture responsives}

La plupart des formules repérées sont donc de type responsif, et cela est vrai tant pour les peu lettrés que par les moins lettrés. Au niveau thématique, elles semblent plus limitées à la mention du courrier et cela est vrai pour tous les scripteurs ; la référence stéréotypée à la santé étant plus représentée chez les peu lettrés, comme cela était d'ailleurs attendu. Voici deux exemples issus des lettres de Lucien et de Joseph :

(11) Je vien de recevoir votre lettre qui m'a bien fais plaisir de vous savoir toujours tous en bonne santé. J'ai reçu une lettre de Marthe et de Marcel, ils vons toujours bien. (Lucien, le 22/02/1916) 
(12) J'ai reçu ta lettre avant-hier. Je suis heureux de te savoir en bonne santé. Le lendemain, j'en ai reçu une de chez nous. (Joseph, le 18/01/1915)

A la parataxe plus simple et linéaire avec laquelle Joseph enchaîne le motif de la santé sur celui de la réception, correspond, chez Lucien, le peu lettré du corpus, une syntaxe plutôt cérémonieuse où l'emploi de subordonnées, qui se fait au détriment de la correction syntaxique, confère au texte une certaine solennité (Bruneton-Governatori, Moreux, 1997). La référence à la santé a ici un statut ambigu : si d'un côté elle rappelle de très près le début de tout échange oral, de l'autre, ainsi énoncée, elle «contribue au caractère lourdement épistolaire du prélude » (Bruneton-Governatori, Moreux, 1997, en ligne). Le thème du courrier peut aussi se décliner sous la forme d'excuses pour un silence prolongé et, par là, la mention de la santé se défige en quelque sorte et est utilisée pour justifier la réponse tardive, comme cela arrive chez Joseph :

(13) Je ne vous ai pas fait réponse plus tôt pour cause de santé là plusieurs jours que j'ai mal aux dents. Hier, J'ai été forcé de me coucher tellement que je souffrais. (Joseph, le 04/02/1915)

Or, si la raison du silence relève d'une motivation personnelle, l'acte de s'excuser et d'en donner une explication est plutôt conventionnel : écrire aux autres membres de la famille devient presque obligatoire en cas de distance géographique, même au-delà des périodes de guerre. Cela est d'autant plus vrai que le fait de s'excuser pour le manque de lettres caractérise même les correspondances bourgeoises du XXème siècle entre les membres d'une même famille (Chotard-Lioret, 1985 : 64), ce qui est confirmé également par la présence de commentaires portant sur l'absence de courrier :

(14) Voilà une dizaine de jours que je n'ai pas reçu une lettre de Vézelay. Vous avez dû recevoir les miennes. Je suis resté à Lunéville 2 jours (Maurice, le $07 / 01 / 1916$ )

Si l'absence de courrier peut même faire l'objet même de reproches de la part des poilus, sa réception est souvent accompagnée d'un sentiment positif. Dans ces cas, l'accusé de réception est alors suivi par l'expression du plaisir éprouvé au moment de la réception par le biais d'une relative rattachée à l'objet « lettre » :

(15) J'ai reçu votre lettre qui m'a bien fais plaisir. Ou don que tu à anvoillé mon sertificat? (Lucien, le 10/03/1916)

Pour ce qui concerne les formules responsives chez les poilus plus lettrés, elles se caractérisent par l'absence de références à la santé et par la réduction de la partie proprement formulaire à l'accusé de réception. Il s'ensuit que ces formules relèvent d'une plus grande simplicité syntaxique : on y trouve soit d'énoncés juxtaposés, comme chez Maurice :

(16) Je viens de recevoir une lettre de Lucien. Ça a l'air d'aller. J'ai aussi reçu une carte de Vincent, qui me dit qu'il est affecté à la $5 \cdot$ section d'infirmiers (Maurice, le 25/1/1915)

(17) J'ai bien reçu le paquet de chocolat et la lettre. J'en assez pour l'instant, il ne faut pas m'en envoyer avant le 1 Décembre. J'ai bien reçu une carte 
postale de quelques mots de Vincent, mais je n'ai pas encore vu sa lettre ni le paquet que vous m'annoncez de lui. J'ai reçu une carte de Marthe m'annonçant (Maurice, le 14/11/1914)

soit d'un énoncé formulaire enchaînant sur une relative ou sur un participe présent en fonction d'épithète, qui n'introduisent plus la seconde partie d'une formule (santé, excuses ou plaisir), mais plutôt des contenus individuels, comme chez Henri :

(18) Je réponds à ta lettre du 21 janvier dans laquelle tu me parles de ton histoire (Henri, le 29/12/1915)

(19) J'ai reçu une lettre de toi, m'annonçant de bonnes choses qui seront les bienvenues, (Henri, le 01/01/1916)

Or, si le modèle formulaire apparaît respecté chez tous les scripteurs du corpus, chez les plus lettrés la seule mention de l'accusé de réception semble remplir essentiellement la fonction d'insérer la lettre en train d'être écrite dans l'échange épistolaire, sans que cela soit reconductible à l'adoption d'un véritable style formulaire.

\subsubsection{Les formules d'ouverture déclaratives}

En ce qui concerne les formules d'ouverture déclaratives, chez les moins lettrés on constate la présence d'un énoncé construit sur un verbe du type envoyer, écrire suivi de constructions syntaxiques permettant de transmettre des nouvelles sur l'état de santé (qui sont toujours rassurantes) et, parfois, d'un deuxième énoncé exprimant le souhait que la santé du destinataire soit bonne. Voici quelques exemples, dont on pourra apprécier les nombreuses micro-variations lexicales et syntaxiques :

(20) Quelques mots pour vous donner de mes nouvelles qui sons toujours bonnes pour le moman (Lucien, le 19/04/1917)

(21) Je vous envoie ces quelques lignes pour vous donner de mes nouvelles qui sont toujours bonnes. Je suis toujours au meme endroit. (Joseph, le 20/12/1914)

(22) Je vous envoi ces quelques mots pour vous donné de mes nouvelles. (Lucien, sans date, 1917)

(23) Vous devez être inquiet de ne pas recevoir de mes nouvelles. Je suis toujours en bonne santé et j'espère que vous êtes tous de même. (Joseph, le 30/07/1914)

D'abord, les variations concernent la présence/absence de la première partie de la formule construite sur le verbe pivot envoyer : dans [20], en effet, il n'est pas explicité, bien qu'il puisse être reconstruit à partir de la présence du complément d'objet direct « quelques mots ». Ensuite, elles portent sur la présence/absence du circonstant temporel «pour le moment », qui ne se retrouve que dans la première formule et de l'énoncé exprimant le souhait pour le destinataire (dans [23], «j'espère que vous êtes tous de même »). Enfin, au niveau lexical, "(ces) quelques mots» dans [20] et [22] peuvent être remplacés par «ces quelques lignes » dans [21], bien que cette seconde option soit largement moins répandue 
dans le corpus. Le figement semble donc plutôt fort, et cela est encore plus évident dans l'exemple suivant, où on a rapporté le texte entier de la très courte lettre :

\section{(24) Je vous envoi ces quelques mots pour vous donné de mes nouvelles}

Je par au frond aujourd'hui au $174^{\circ}$ de ligne. (Lucien, sans date, 1917)

Les « nouvelles» annoncées par la formule ne sont finalement qu'une (le départ au front) : le caractère fortement stéréotypé de la formule adoptée montre clairement qu'il n'y a pas d'adaptation de la partie formulaire au contenu qui la suit. Il en va autrement pour les formules déclaratives des scripteurs plus lettrés, comme cet exemple le montre :

(25) Ce n'est plus un homme qui vous écrit, c'est un bandit à longue barbe, qui couche dehors toutes les nuits, crotté, boueux, ne changeant jamais de linge ni de chaussures, mangeant ce qu'il trouve, des bouts de biscuit et un peu de conserve et restant souvent 24 heures sans trouver quoi que ce soit. (Henri, le 17/09/1914)

S'il semble difficile de parler de formule, il est vrai néanmoins qu'Henri inscrit en début de sa lettre un énoncé explicitant l'acte d'écrire à sa femme, tout comme cela arrive chez les scripteurs moins lettrés. Du point de vue fonctionnel, cet énoncé n'apparaît pas très loin des formules plus stéréotypées des peu lettrés : les deux permettent aux scripteurs d'entrer dans l'écrit, bien que la représentation de l'acte d'écriture chez Henri soit fortement individualisée. Cela est évident tant au niveau sémantique, qui porte sur l'état d'âme ressenti par le scripteur qu'au niveau syntaxique : la structure présentative «c'est » jalonne tout le premier paragraphe, sans qu'il soit possible de délimiter de manière précise le passage de la partie introductive à la phase individuelle. Chez Maurice aussi on rencontre des variations, bien que le moule formulaire soit plus visible :

(26) Je me suis dépêché ce matin de vous griffonner quelques mots pour vous donner immédiatement ma nouvelle adresse car le vaguemestre ne fait qu'une levée par jour, le matin à $7 \mathrm{~h}$. Donc, hier nous avons été affectés (Maurice, le 17/4/1915)

Les variations par rapport aux formules des moins lettrés concernent ici surtout le lexique, avec l'emploi des lexèmes plus expressifs «dépêché » et «griffonner »qui insistent sur la rapidité de l'acte d'écriture en train de se faire. Chez les scripteurs plus lettrés, on peut rencontrer aussi la référence à la santé, mais l'on assiste dans ces cas à un certain défigement de la formule :

(27) Ça va, suis en bonne santé. Depuis 10 jours, on marche jour et nuit. (Maurice, le 11/09/1914)

Dans cet exemple, la formule rappelle de très près l'ouverture d'une conversation en face-à-face et ne subit pas l'effet de scripturalité montré par la mention de la santé dans les ouvertures des peu lettrés : il n'y a là aucune volonté d'anoblir le style d'écriture, ce qui est évident aussi dans l'énoncé qui suit, où le scripteur omet le pronom personnel sujet et, par là, simule presque un échange oral avec ses destinataires. Il arrive aussi que, contrairement à ce qu'on a pu constater dans les ouvertures des scripteurs peu lettrés, la partie formulaire 
soit adaptée, reprise ou justifiée dans la suite du corps de la lettre, comme dans le cas suivant :

(28) Je suis toujours en bonne santé et depuis le 8 septembre, j'attends une lettre de vous. Je commence à trouver le temps long, vous devez le comprendre. (Maurice, le 21/10/1914)

On constate là l'intégration de la partie formulaire (l'attente de la lettre) dans le corps du texte à travers une sorte de justification : la monotonie de la vie en guerre semble peser sur le soldat pour qui la lettre représente un moment de distraction compensant les longues heures passées sans rien faire dans la les tranchées. Dans ces exemples, le défigement syntaxique et lexical montre un certain degré d'appropriation du discours formulaire de la part des scripteurs et une meilleure intégration des rituels d'ouverture dans le corps du texte.

\section{Les transitions}

Comment passent les scripteurs de la formule d'ouverture au corps du texte ? L'analyse du corpus montre que dans plus que la moitié du corpus analysé (57\%), les formules ritualisées d'ouverture ne sont pas intégrées dans le corpus de la lettre par des transitions. Et cela est vrai surtout pour les peu lettrés, alors que chez les plus lettrés les pourcentages diminuent sensiblement (Henri, 3\% ; Maurice, 37\%, Joseph, 75\% ; Lucien, 50\%). Voici deux exemples caractérisés par l'absence de transition :

(29) J'ai reçu votre lettre ce matin. Je ne suis pas encore hopairé, ça va aitre pour mercredi. Je ne pense pas que ça me fasse bien mal. (Lucien, le 24/01/1916)

(30) J'ai reçu votre lettre hier soir, je vous fait réponse aussitot. Je suis dans la Somme à $15 \mathrm{~km}$ d'Ambleville. Je suis très bren. (Joseph, le 20/02/1915)

Une coupure typographique forte représentée par le point suffit aux scripteurs pour passer au corps de la lettre sans qu'ils ressentent la nécessité d'intégrer davantage la formule dans leur texte. Les scripteurs les moins lettrés du corpus (Joseph, Lucien) semblent utiliser moins de transitions que les scripteurs les plus lettrés (Henri, Maurice) : l'élément formulaire est mieux intégré dans le corps de la lettre chez les plus lettrés. Dans la suite on montrera les moyens langagiers utilisés par les scripteurs dans la transition entre la forme rituelle d'ouverture et le corps de la lettre à partir des cas les plus représentatifs du corpus.

\subsection{Transition par rection}

Sur la base du modèle de transitions élaboré par Große S., Steuckardt A., Dal Bo B., Sowada L. (2016) et des études sur les « rapports de rection » du Groupe de Fribourg (2012 : 47), on peut identifier des transitions «par rection» où la formule est suivie d'un "constituant facultatif», représenté par un verbe métacommunicatif sous la forme de circonstant ou de construction épithète régissant un objet. Cet objet peut relever de la 
dimension individuelle ou rituelle. Dans ce second cas, il sera coordonné à un second objet de nature individuelle. La transition par rection est certainement le cas le plus fréquent de transition dans les lettres et semble caractériser de manière plus nette les lettres responsives des plus lettrés. Un seul cas a été relevé chez une lettre déclarative sous la plume de Lucien :

(31) Je vous anvoie ces quelque mots pour vous dirre que je m'an va Samedi ou Lundi. (Lucien, le 25/02/1916)

La formule ritualisée d'ouverture déclarative est ici suivie par une text-structural formula "pour vous dire que » introduisant bien un objet individuel (l'annonce du départ). Cela dit, la plupart d'occurrences de ce type de transition se trouve dans les lettres responsives. L'accusé de réception est alors suivi d'une text-structural formula qui peut se présenter sous la forme d'épithète de l'objet «lettre », à travers un participe présent ou un gérondif :

(32) J'ai reçu une lettre de toi, m'annonçant de bonnes choses qui seront les bienvenues, car il est difficile de s'approvisionner ici. (Henri, le 01/01/1916)

(33) J'ai reçu une lettre de Charles an me disans qu'il étais demi-porsion (Lucien, le 24/05/1918)

Chez les plus lettrés, la transition est plus volontiers assurée par une relative qui permet de reprendre le contenu de la lettre reçue :

(34) Je réponds à ta lettre du 21 janvier dans laquelle tu me parles de ton histoire, de Boileau, etc ... J'aime à ce que tu me parles du cours, de ce que vous apprenez. Cela me rappelle l'heureux temps où je t'ennuyais tant pour que tu aies un bon rang en classe. Ici, nous sommes depuis quelques jours toujours en mouvement. (Maurice, le 13/02/1917)

(35) J'ai reçu hier $\mathbf{2 0}$ janvier ta lettre du 17 dans laquelle tu me parles du dessus de piano d'Adèle et où se trouve le petit mot de Jeannette. Aujourd'hui jeudi il pleut toute la journée et tout s'est tu. (Henri, le 21/01/1915)

Dans ces exemples, on constate la présence du même schéma textuel : formule réduite à l'accusé de réception, constituant facultatif introduisant le contenu de la lettre reçue et, enfin, déictique spatial («Ici ») ou temporel («Aujourd'hui ») qui permet d'introduire de nouvelles informations que le scripteur veut transmettre dans la lettre en train de se faire et qui marque l'ancrage du scripteur dans la situation d'énonciation spécifique. Bien que la reprise des contenus de la lettre contienne évidemment des éléments individuels, l'organisation textuelle apparaît plutôt figée, de sorte qu'on puisse presque considérer la reprise du discours de la lettre précédente comme une phase intermédiaire entre le rituel d'ouverture et le véritable corps de la lettre, marqué par le changement de perspective du scripteur. 


\subsection{Transition par les déictiques}

Le propos individuel peut aussi être introduit par un déictique spatial ou temporel ou par un présentatif qui permet au scripteur d'ancrer son point de vue dans la situation de rédaction et, par là, d'introduire le propos individuel :

(36) Je suis toujours en bonne santé et j'espère que vous êtes tous de même. Voilà le temps qui commence à se rafraîchir. Ce matin, il y avait de la gelée blanche, mais dans la journée, il fait beau (Joseph, le 26/09/1914)

(37) J'ai reçu hier soir vos lettres respectives et leur contenu ; me voici avec du pain sur la planche; le colis annoncé me parviendra certainement ce soir. Le service... (Maurice, le 28/04/1915)

Comme ces exemples le montrent, il peut s'agir des présentatifs « voilà » et «voici » régissant soit un groupe nominal soit une prédication par le biais d'une relative. Ces présentatifs contribuent d'ailleurs à inscrire en discours un certain effet d'oralité, tant il est vrai que « cette structure est fréquemment employée à l'oral, car elle sert à désigner un référent dans la situation d'énonciation » (Riegel et alii., 1994 : 453), ce qui est montré par le fait qu'ils sont souvent accompagnés d'un acte de monstration supposant la présence simultanée du locuteur et de l'interlocuteur. On peut remarquer, entre autres, dans le premier exemple, la présence du démonstratif «ce» rattaché à l'expression du temps ( «matin ») qui sert à introduire un énoncé qui spécifie davantage le thème introduit par le présentatif : on voit là bien une progression thématique linéaire, qui va du général et rituel au particulier et individuel. A peu près la même fonction est remplie par des déictiques temporels et spatiaux: eux aussi, ils permettent d'inscrire dans la lettre la situation d'énonciation dans laquelle se trouve le soldat:

(38) J'ai reçu une letre de Joseph hier q[u']elle était datée du janvier Il me dit qu'il avait quitté la Belgique en Décembre a présant j'ai touché l'argent de mon linge g'ai touché 15 frs (Lucien, le 08/01/1915)

(39) Suis en bonne santé. Pour l'instant, c'est un peu plus calme. On couche toujours (Maurice, le 29/09/1914)

(40) J'ai reçu ta première lettre vendredi et deux lettres de Maurice. Ici, les régiments sont partis (Henri, le 10/08/1914)

Ces déictiques permettent ainsi aux scripteurs de passer du rituel à l'individuel, bien que de manière quelque peu abrupte, par l'inclusion du destinataire dans la situation de rédaction du scripteur.

\subsection{Transition par coordination}

Dans un nombre exigu de cas, la transition entre le rituel et l'individuel se fait par une simple coordination se situant à la fin de la formule. Dans la plupart des exemples, c'est la conjonction « et » qui est utilisée : 
(41) J'ai bien reçu votre lettre. Je suis heureux de vous savoir tous en bonne santé. Je suis toujours en bonne santé et au même endroit. (Joseph, le $08 / 03 / 1915)$

Le scripteur relie l'information sur le lieu où il se trouve à l'élément formulaire qui est, dans ce cas, plutôt long et composé de plusieurs parties (accusé de réception + réjouissance pour le bon état de santé du destinataire + expression de son propre état de santé), typique des rituels épistolaires des peu lettrés. Cela dit, la conjonction introduisant un élément individuel peut aussi enchaîner sur la formule réduite à l'accusé de réception, comme cela arrive sous la plume de Maurice :

(42) J'ai bien reçu votre lettre du 27 et suis content de savoir que Lucien a été en permission. (Maurice, le 03/01/1916)

La conjonction peut être aussi représentée par « car » :

(43) J'ai bien reçu votre je n'ai pas eu le temps de vous répondre plutôt, car le soir, nous arrivons très tard et partons de bonne heure le matin. (Joseph, le $16 / 09 / 1914)$

Les excuses pour le silence prolongé qui déclinent le motif de l'accusé de réception sont ici justifiées par le scripteur, ce qui permet une meilleure intégration de l'élément formulaire dans le corps du texte.

\subsection{Transition par la structure informationnelle}

La transition peut aussi s'effectuer par le biais de l'introduction d'un nouveau topique. Pour ce faire, les scripteurs de ce corpus recourent à deux moyens principaux, à savoir les constructions pseudo-clivées et les marqueurs de topicalisation, cette seconde catégorie étant largement plus représentée que la première. Voici un exemple de construction pseudoclivée utilisée comme élément de transition :

(44) J'ai reçu votre lettre hier. Je suis heureux de vous savoir en bonne santé ce qui me fait le plus plaisir, c'est de savoir Marcel au repos. (Joseph, le $18 / 02 / 1915)$

Ce type de construction, typique de l'oral (Blanche-Benveniste, 2000), permet d'extraire un élément de l'énoncé pour le placer dans une position rhématique. Dans ce cas, le scripteur extrait l'élément formulaire du plaisir éprouvé pour l'information sur l'état de bonne santé de ses destinataires pour le spécifier et le délimiter dans la seconde partie de la pseudo-clivée identificative (Apotheloz, Roubaud, 2015) : la première proposition de la pseudo-clivée réactive l'objet de discours rituel apparu antérieurement («Je suis heureux de vous savoir en bonne santé ») et permet au scripteur de le focaliser dans la seconde proposition pour l'adapter à ses propos individuels. Si cette construction n'est pas très fréquente dans le corpus, les transitions de structure informationnelle se font plus volontiers par le biais d'un marqueur de topicalisation, où on peut rencontrer quant à moi et vers moi:

(45) J'ai bien reçu votre lettre. Je suis content de vous savoir tous en bonne santé. Quant à moi, c'est toujours pareille. (Joseph, le 18/04/1915) 
(46) J'ai reçu la lettre du 14 et une de Marcel du 14 Il me disais qu'il partais en permission le 15 d'après ce qu'il ce passe, je ne croi pas qu'il soit partis. Vers moi, sa barde aussi pour le moman (Lucien, le 18/07/1918)

Comme le rappellent Große S., Steuckardt A., Dal Bo B., Sowada L. (2016), la valeur énonciative de ces marqueurs est celle «d'introduire un nouveau topique ou de rappeler la présence d'un topique supposé connu parce qu'il s'agit d'une correspondance suivie » (en ligne) : dans ces exemples, ils assurent le passage du rituel ou semi-rituel (dans le second exemple) à l'individuel et semblent caractériser davantage l'écriture des peu lettrés. Ce bref tour d'horizon sur l'éventail des stratégies adoptées par les scripteurs pour passer des éléments rituels de l'ouverture des lettres au corps du texte, permet d'identifier certaines tendances caractérisant l'écriture des peu lettrés et des plus lettrés. Il semble en effet que les éléments rituels chez les peu lettrés sont moins intégrés dans le corps de la lettre que chez les plus lettrés : la plupart des lettres de Lucien et de Joseph ne présentent aucune marque de transition, qui se fait soit par la ponctuation (chez Lucien) soit par la typographie (le passage à la ligne chez Joseph). Seulement dans un nombre limité d'exemples on observe la présence de constructions pseudo-clivées (et on voit là sans doute l'influence de l'oral) et de marqueurs de topicalisation (dont le prototypique quant à moi). Les scripteurs plus lettrés (Maurice et surtout Henri) semblent privilégier la transition par rection qui présuppose un continuum syntaxique plus fort entre le rituel et l'individuel et, par là, un degré d'appropriation de la formule plus important. Cette interprétation est supportée par le fait que chez ces scripteurs, l'élément formulaire est souvent suivi d'une phase qu'on peut qualifier d'intermédiaire, qui porte sur les contenus de la lettre reçue et qui permet de passer de manière progressive à l'élément individuel. Cela est bien visible aussi dans les cas où les scripteurs recourent aux déictiques et aux présentatifs (à présent, ici, voici, voilà) qui permettent aussi de réduire la distance énonciative entre le scripteur et son destinataire.

\section{Les clôtures}

Lieux privilégiés de l'expression des affects (Branca-Rosoff, 1997), les clôtures des lettres présentent un certain figement au niveau structurel : il s'agit là plus de routines discursives qui sont reprises par les scripteurs, de modèles sous-jacents à tout un ensemble de productions individuelles caractérisées par des variations thématiques et structurelles qui empêchent l'identification d'un modèle unitaire. Néanmoins, il semble que la plupart des clôtures soient caractérisées par une structure bipartite qui prévoit un premier énoncé annonçant la clôture et un second énoncé constitué de la véritable formule de congé, ces deux énoncés pouvant accueillir des contenus sémantiques un tant soit peu différents et se réaliser par des moyens syntaxiques variés. Comme on a déjà pu le constater (cf. tableau 4), les formules de clôture semblent caractériser davantage l'écriture des scripteurs plus lettrés du corpus, à savoir Henri et Maurice, chez qui ces formules sont identifiables dans la presque totalité des lettres analysées, alors qu'elles seraient beaucoup moins fréquentes chez les scripteurs les moins lettrés.

\subsection{Enoncé métacommunicatif et formule de congé}


Selon ce modèle, le premier énoncé est constitué d'un performatif métacommunicatif annonçant la clôture de la lettre et le second est représenté par la formule de congé proprement dite. Voici quelques exemples :

(47) Je termine et vous embrasse bien affectueusement. Votre Maurice. (Maurice, le $5 / 1 / 1916)$

(48) Je termine en vous envoyant de la lisière du Bois-Le-Prètre les meilleur baisers de votre pioupiou. Maurice. (Maurice, le 28/4/1915)

(49) Je vous quitte en vous embrassant bien affectueusement comme je vous aime. (Joseph, le 8/1/1917)

Le premier énoncé «je termine» peut être considéré comme une formule de clôture informative ou pré-clôture qui est typique des échanges oraux ordinaires (KerbratOrecchioni, 1998), alors qu'elle est plus rare dans les correspondances littéraires. Cet énoncé est constitué d'un performatif métacommunicatif qui annonce la fin de l'acte de communication (qu'il s'agisse de l'acte d'écriture : je termine ou de l'acte de terminer un échange oral : je vous quitte) et il est suivi d'une transition, assurée soit par une coordination soit par une forme en -ant, introduisant la formule de congé. Les variations concernent tant la première partie que la seconde. Une première distinction lexicale concerne, comme les exemples ci-dessus le montrent, l'emploi de terminer [47] et [48] ou de quitter [49], l'un pointant la dimension de l'écriture, l'autre celle de l'oral, dans la formule informative qui ouvre la voie à l'expression de l'affectivité dans la formule de congé : on pourra donc embrasser son destinataire, élargir le destinataire de l'acte affectueux à l'ensemble de la famille ou envoyer des baisers. Embrasser est souvent suivi d'un modalisateur adverbial montrant davantage la relation affective entre destinateur et destinataire. Les exemples montrent aussi que le moule formulaire accepte des expansions tant internes («de la lisière du Bois-Le-Prètre ») qu'à droite («de votre pioupiou», «comme je vous aime»), qui permettent aux scripteurs de s'approprier la formule et d'ajouter des éléments individuels susceptibles de mieux ancrer l'objet rituel dans leur ressenti. Cet effort d'appropriation de l'élément rituel est révélé surtout dans [48], qui présente les deux types d'expansions, interne et à droite: il relie la signature, fort personnalisée («votre pioupiou»), à la formule de congé, sous forme de complément précédé de «de». Ce dernier ajout se fait en dépit du respect de la norme linguistique et crée un effet de redondance et d'insistance sur la présence du sujet énonciateur dans son énoncé. L'espace entre le performatif et la formule de congé peut aussi accueillir des informations individuelles qui atténuent de quelque sorte la performativité de la formule de clôture informative :

(50) Je m'arrête parce que je continuerais ainsi jusqu'à dire des bêtises. Mais je vous assure que j'en ai marre, marre, marre !!! Je vous embrasse tous quatre de tout cour, Votre Maurice. (Maurice, le 01/01/1916)

Le performatif n'est pas suivi d'effet, il est de quelque sorte raté : le scripteur ne termine pas vraiment sa lettre là où il l'a annoncé. Il y trouve l'espace nécessaire pour justifier la clôture de la lettre et pour exprimer son état d'âme avant de prendre définitivement congé avec l'expression de l'affectivité élargie à l'ensemble des membres de sa famille. 


\subsection{Annonce de clôture et formule de congé}

Surtout chez les plus lettrés, le performatif typique de la formule de clôture informative peut commuter avec un énoncé remplissant la même fonction d'annonce de la fin de la lettre, mais qui porte sur le manque de sujets ultérieurs à communiquer au destinataire. En voici deux exemples issus de la correspondance d'Henri :

(51) Voilà tout ce que j'ai à vous dire. Le 36e actif part demain mercredi pour Laon. Les hommes y vont de bon cœur et seraient très peinés si l'ordre venait de démobiliser. Ecrivez-moi à l'adresse suivante : Commandant de réserve au 36e, Caen. Je vous embrasse, Henri (H.B., le 4 aout 1914)

(52) Voilà tout ce qu'il y a de nouveau ici. Drôle de guerre où l'on en est réduit à parler de cuisine. Je vous embrasse. Henri (H.B., le 21/01/1915)

L'énoncé métacommunicatif est introduit chaque fois par le présentatif voilà, dont la valeur résomptive lui permet d'assurer la cohésion textuelle (Col G., Danino C., Rault J. et Knutsen D., 2016, Oppermann-Marsaux, 2006, Rabatel, 2000). Or, comme on peut le constater, l'annonce de clôture n'est jamais suivie de la formule de congé : le scripteur rajoute encore quelques dernières informations libres, comme s'il n'arrivait pas à respecter le plan annoncé. Et cela est encore plus évident dans l'exemple suivant :

(53) Plus rien à vous dire. Pour le soldat que vous logez, je crois que vous feriez bien de vous en débarrasser. C'est un fricoteur qui manque à sa compagnie, car les soldats ne doivent pas quitter leur escouade. Inventez une histoire. Dites que vous vous absentez ou que vous attendez quelqu'un. Je vous embrasse tous. Henri. (Henri, le 20/8/1914)

Bien que l'inscription dans le texte de la formule de pré-clôture montre une certaine conscience de la structure du texte épistolaire et de sa cohésion interne, Henri semble avoir du mal à respecter ses mêmes propos : le flux verbal prend le dessus et le scripteur n'hésite pas à rajouter des informations qu'il ne ressent pas sans doute comme appartenant au corps du texte proprement dit. L'ajout d'une partie libre peut également s'étendre au-delà de la signature finale, comme dans l'exemple suivant :

(54) Voilà l'heure de se coucher et je vais m'arrêter là. Vous enverrez cette lettre à René ; je vais lui écrire un petit mot, mais ce serait trop long de lui dire tout cela. Bonsoir et à tous de grosses, grosses mignonnes de votre Maurice. P.S. Au moment où je vais me coucher et terminer, on annonce la capture d'un espion capitaine d'artillerie qui, à la dernière attaque du 16ge, avait fait tirer sur nos lignes, il faisait des signaux et repérait nos batteries, il a été lynché par les Mussipontains. (Maurice, le 24/4/1915)

Dans cet extrait, voilà ne joue pas seulement sa fonction résomptive: il permet également un changement de perspective de l'énonciateur qui inclut, par là, le destinataire dans le hic et nunc de la «conversation » épistolaire (« voilà l'heure de se coucher »), ce qui est confirmé par la reprise anaphorique de la situation d'énonciation par le biais de la particule adverbiale «là », dans l'énoncé performatif qui suit. D'ailleurs, ce performatif n'est pas, encore une fois, suivi d'effet : cet énoncé ne permet pas vraiment de clôturer le 
texte, qui enchaîne sur des informations libres suivies non seulement de la formule de congé mais aussi d'un post scriptum plutôt long qui permet lui aussi d'inscrire le destinataire dans la situation de rédaction ( $\mathrm{Au}$ moment où je vais me coucher »), en agissant du côté du dédoublement du contexte d'énonciation et de la réduction de la distance énonciative à la fois. C'est ainsi que le scripteur ressent sans doute le besoin de réaffirmer l'acte de terminer sa lettre par la reprise sous forme infinitive ( « et terminer ») du performatif plus stéréotypé énoncé plus haut. En cas d'absence de voilà initial, l'ancrage dans la situation d'énonciation du scripteur peut être assuré par la présence d'un déictique temporel, comme dans l'exemple suivant :

(55) Je n'ai pas le temps de vous écrire plus longuement aujourd'hui. À bientôt d'autres nouvelles. Mille baisers de votre Maurice. (Maurice, le $17 / 4 / 1915)$

Dans cet extrait, ce n'est plus le manque de mots qui est mis en avant par Maurice pour justifier la clôture de sa lettre : c'est le moment d'énonciation lui-même (le manque de temps) qui fonctionne comme justification de l'annonce de fin de la lettre. Entre la formule de clôture informative et la formule de congé, Maurice inscrit le thème des salutations qui caractérise un certain nombre de variantes formulaires, comme on le verra dans la suite.

\subsection{De quelques variations thématiques}

La structure bipartite des clôtures admet un certain nombre de variantes, surtout au niveau sémantique. En effet, l'une des deux parties formulaires peut porter sur la santé du scripteur ou sur celle d'une personne connue par le destinataire, comme dans les extraits suivants

(56) Je me porte bien et vous embrasse tendrement. (Henri, le 20/09/1914)

(57) Marthe est en bonne santé. Elle vous embrasse ainsi que moi (Maurice, le $25 / 05 / 1917$ )

(58) Ge ne voit plut granchose à vous dire je suis toujours en bonne santé j'espaire que vous êtes tous de même (Lucien, le 8/1/1915)

Ou sur des renseingnements sur le courrier et sur la correspondance : ce thème peut alors se décliner sous la forme d'une attente d'une réponse ou d'une demande plus directe de courrier :

(59) J'attends de vos nouvelles et vous embrasse tous (Maurice, le 20/08/1914)

(60) Il faudrai mécrire de tan en tan me raconté tes explois (Lucien, le $17 / 02 / 1915)$

Un dernier topos est représenté également par les salutations adressées par le destinateur à d'autres personnes du village (ce qui confirme la dimension presque publique de la lettre) ou par les salutations adressées aux destinataires de la part de personnes connues vivant au front, avec le scripteur. Ces salutations ont l'effet de tisser des liens communautaires, de montrer la participation de l'énonciateur au réseau social d'origine : 
(61) Je viens de recevoir une lettre de Marthe. J'ai reçu aussi une carte de Joseph. Savelly et Grossin vous envoient le bonjour (Maurice, le 10/01/1915)

(62) Tante Célénie vas toujours bien tu lui soitra le bonjours pours moi. Tu soitras le bonjours à Brisdoux pour moi. Je termine je vais allé me faire piqué l'épaule. (Lucien, le 12/1/1915)

Ce dernier exemple montre encore un cas d'inclusion du destinataire dans la situation d'énonciation du scripteur qui, par là, réduit la distance énonciative avec l'autre pôle de l'échange épistolaire. Par l'inclusion du destinataire dans leur situation d'énonciation à travers les déictiques et les présentatifs, par une certaine redondance sémantique (cf. le cas de "comme je vous aime ») et par l'ajout d'expansions internes ou à droite des éléments formulaires («bien tendrement », «bien affectueusement », « de tout cœur », les scripteurs tentent de réduire la distance, énonciative mais aussi physique semblerait-il, entre eux et leurs destinataires, d'exprimer leur affectivité au-delà de la formule stéréotypée, qui ne suffit pas toujours à remplir le besoin de communication des scripteurs. Le moule formulaire semble donc accueillir, bien plus que dans les ouvertures, des variations thématiques et syntaxiques permettant aux scripteurs de faire ressortir l'individuel, ou mieux, une dimension intersubjective, une communion de perceptions, de sens et d'affects avec la ou les personnes aimées ou lointaines.

\section{En guise de conclusion}

$\mathrm{Au}$ terme de cette étude exploratoire, on peut constater que tous les scripteurs semblent recourir aux formules, qu'il s'agisse de celles d'ouverture ou de celles de clôture. Les différences résident plutôt dans leur emploi et dans leur degré d'intégration dans le corps de la lettre. Le fait que la plupart des formules d'ouverture sont repérables dans les lettres des moins lettrés semble confirmer l'hypothèse que la fonction principale de ces rituels consiste en la facilitation de l'entrée dans l'écrit de la part de scripteurs qui n'ont pas de familiarité avec l'écriture. Cette interprétation semble confirmée par le fait que les formules d'ouverture chez les plus lettrés ne participent pas de la construction d'un effet de scripturalité, tel qu'il caractérise les ouvertures des peu lettrés : la syntaxe y est en effet moins cérémonieuse et elles révèlent davantage une certaine influence de l'oralité (çava en début de lettre). Les exemples analysés montrent en effet que l'emploi des formules chez les moins lettrés est souvent accompagné de l'utilisation d'une certaine complexité syntaxique que les scripteurs ne savent pas toujours trop maîtriser. Cela peut sans doute être lié au statut semi-public des lettres et à l'importance conférée par les scripteurs à ces lettres, considérées comme moyens pour préserver les rituels sociaux de la communauté d'origine. Les clôtures semblent caractériser davantage l'écriture des scripteurs plus lettrés: les formules y sont plus nombreuses et plus codées que chez les moins lettrés. Cela dit, comme on a pu le constater, le moule formulaire, plus ou moins respecté par tous les scripteurs plus lettrés dans les conclusions, n'empêche pas aux scripteurs d'y insérer des variations individuelles qui font de ces clôtures le lieu où ressort l'affectivité et où se joue la relation avec le/les destinataire/s. Les scripteurs plus lettrés s'approprient ces formules, tout comme le montrent aussi les transitions analysées: les formules sont réduites et adaptées au contenu qui suit, contrairement à ce qui se passe chez les peu lettrés. Néanmoins, il me 
semble que la présence massive de formules chez tous les scripteurs du corpus permet d'avancer l'hypothèse, à vérifier sur des corpus plus larges, de l'existence d'une macrostructure plus ou moins abstraite et sous-jacente à l'ensemble des lettres des moins aux plus lettrés, qui intègre patrons morphosyntaxiques avec des topiques récurrentes et qui ne serait pas utilisée exclusivement par des scripteurs non lettrés.

\section{Références bibliographiques}

Apotheloz, D., Roubaud, M.-N.. (2015). Constructions pseudo-clivées. Encyclopédie Grammaticale $d u$ Français, 2015. En ligne: https://halshs.archives-ouvertes.fr/hal01542097/document, consulté le 11/12/2017.

Bellosi, G., Savini, M. ([2002] 2014). Verificato per censura. Lettere di soldati romagnoli nella prima guerra mondiale. Cesena : Società Editrice « Il Ponte Vecchio ».

Bénard, H.(1999). De la mort, de la boue, du sang. Lettres de guerre d'un fantassin de 1418. Paris : Grancher.

Branca-Rosoff, S. (1990). Conventions d'écriture dans la correspondance des soldats. Mots, 24, 21-36.

Branca-Rosoff, S. (2015). Rituels épistolaires ou flux verbal. Deux formes d'appropriation de l'écriture. Steuckardt, A. (dir.). Entre village et tranchées. L'écriture de Poilus ordinaires. Uzès : Inclinaison, 41-52.

Bruneton-Governatori, A., Moreux B., (1997). Un modèle épistolaire populaire. Les lettres d'émigrés béarnais ». Fabre D. (dir.). Par écrit. Ethnologie des pratiques d'écriture quotidiennes. Paris: Maison des Sciences de l'Homme, 79-103. En ligne: http://books.openedition.org/editionsmsh/3957, consulté le 11/12/2017.

Chartier, R. (1991). Des « secrétaires » pour le peuple ? Les modèles épistolaires de l'Ancien Régime entre littérature de cour et livre de colportage ». Chartier R. (éd.), La Correspondance. Les usages de la lettre au XIXe siècle, Paris : Fayard, 159-207.

Chotard-Lioret, C. (1985). Correspondre en 1900, le plus public des actes privés ou La manière de gérer un réseau de parenté. Ethnologie française, 15, 63-72.

Col G., Danino, C., Rault, J. et Knutsen, D. (2016). Rôle de voilà dans l'affirmation : valeur confirmative et marque d'intégration d'informations. Testi e Linguaggi, Università di Salerno. En ligne : https://hal.archives-ouvertes.fr/hal-01377898, consulté le $11 / 12 / 2017$.

Gibelli, A. (1991). L'officina della guerra. La Grande Guerra e le trasformazioni del mondo mentale. Torino : Bollati Boringhieri.

Große, S. (2011). L'évolution des manuels épistolographiques français en tant que traités normatifs. Branca-Rosoff S., Fournier J.-M., Grinshpun Y., Régent-Susini A. (dir.). Langue commune et changements de norme Paris : Champion, 239-252. 
Große, S., Steuckardt, A., Dal Bo, B., Sowada, L. (2016). Du rituel à l'individuel dans des correspondances peu lettrées de la Grande Guerre. Actes du 5e Congrès Mondial de Linguistique Française. En ligne: https://www.shsconferences.org/articles/shsconf/abs/2016/05/shsconf_cmlf2016 06008/shsconf_cmlf20 16 06008.html, consulté le 11/12/2017.

Hébrard, J. (1991). La lettre représentée. Chartier R. (éd.), La Correspondance. Les usages de la lettre au XIXe siècle. Paris : Fayard, 279-365.

Kerbrat-Orecchioni, C. (1998). L'interaction épistolaire. Siess, J. (dir.). La lettre entre réel et fiction. Paris : SEDES, 15-36.

Luxardo, G., (2015). Fréquences des colis et marmites : comment mesurer la languitude?. Steuckardt A. (dir.). Entre village et tranchées. L'écriture de Poilus ordinaires. Uzès : Inclinaison, 113-123.

Moreux, B. (2001). Un aspect de la culture des peu-lettrés: la lettre familiale populaire. Poueyto J.-L. (dir.). Illetrismes et culture, Paris, L'Harmattan, p. 117-129.

Oppermann-Marsaux, E. (2006). Les origines du présentatif voici/voilà et son évolution jusqu'à la fin du XVIème siècle. Langue française, 149, 77-91.

Pellegrini, F. (2015). Logiques épistolaires. Steuckardt A. (dir.). Entre village et tranchées. L'écriture de Poilus ordinaires. Uzès : Inclinaison, 101-112.

Rutten, G., van der Wal, M. J. (2013). Epistolary formulae and writing experience in Dutch letters from the seventeenth and eighteenth centuries. Van der Wal, M. J., Rutten, G. (dir.). Touching the Past. Studies in the historical sociolinguistics of ego-documents. Amsterdam / Philadelphia : Benjamins, 45-66.

Papillon, L. (2003). "Si je reviens comme je l'espère ». Lettres du Front et de l'Arrière. 1914-1918. Paris : Grasset.

Pensuet, M. (2010). Écrit du front, Lettres de Maurice Pensuet, 1915-1917. Paris : Tallandier.

Rabatel, A. (2000). Valeurs représentative et énonciative du « présentatif » c'est et marquage du point de vue. Langue française, 128, 52-73. 